\title{
Distribution of cervical lesions in high-risk HPV (hr-HPV) positive women with ASC-US: A retrospective single-center study in China
}

\author{
Zhiling Wang \\ Qilu Hospital of Shandong University
}

Ying Gu

Qilu Hospital of Shandong University

Hui Wang

Qilu Hospital of Shandong University

Junyu Chen

Qilu Hospital of Shandong University

Yawen Zheng

Qilu Hospital of Shandong University

Lin Jia

Qilu Hospital of Shandong Unversity

Baoxia Cui

Qilu Hospital of Shandong University

Xingsheng Yang ( $\nabla$ xingshengyang@sdu.edu.cn )

Qilu Hospital of Shandong University https://orcid.org/0000-0002-4946-2033

\section{Research article}

Keywords: uterine cervical neoplasms, Human Papillomavirus, atypical squamous cells of undetermined significance, cytology

Posted Date: September 11th, 2019

DOI: https://doi.org/10.21203/rs.2.14278/v1

License: @ (i) This work is licensed under a Creative Commons Attribution 4.0 International License. Read Full License

Version of Record: A version of this preprint was published at Virology Journal on November 23rd, 2020. See the published version at https://doi.org/10.1186/s12985-020-01455-2. 


\section{Abstract}

Objective To investigate distributions of cervical lesions and factors associated with the severity of the cervical lesions in high-risk HPV (hr-HPV) positive women with ASC-US cytology.

Methods Clinical information of 250,000 women who underwent HPV and cytological test was collected from January 2012 to January 2019. The association between the severity of the cervical lesions and hr-HPV genotypes, hr-HPV viral load, and ages, were analyzed in hr-HPV-positive/ASC-US women.

Results 3459 hr-HPV-positive/ASC-US women were enrolled in this study. Overall, $43.51 \%$ of women with ASC-US had normal histological results, $34.35 \%$ had high-grade squamous intraepithelial lesion (HSIL), and $1.30 \%$ had cervical cancer. The rate of HSIL or worse (HSIL+) in women with single HPV16 infection (63.09\%) was the highest, followed by HPV33 (57.50\%), HPV51 (36.11\%), HPV58 (36.11\%), HPV52 (28.28\%), HPV18(26.37\%), HPV66 (19.35\%), HPV39 (18.92\%), HPV53 (15.00\%), and HPV56 (8.51\%). Detection rate of HSIL+ in low, intermediate and high viral-load groups were 15.87\% ( $\mathrm{n=30}$ ), $34.91 \%(n=74)$ and $40.68 \%(n=214)$ (Cochran-Armitage Trend test $\chi 2=35.03, P<0.0001)$. Compared with the 51-60-year-old group (21.65\%), the women in $\leq 30$ (40.52\%), 31-40 (39.67\%), and 41-50 (34.22\%) year-old groups had significantly higher risk of HSIL+. The women in $\leq 51-60(2.68 \%)$ and $>60$ (3.41\%) year-old groups were at increased risk for cervical cancer, compared with the $\leq 30$-year-old group $(0.61 \%)$.

Conclusion ASC-US women with HPV 16/18/33/51/52/58 single infection and multiple infections, as well as high HPV viral loads, have high risk of HSIL+. Young women and older women have higher HSIL+ detection rates, while the latter has a higher cervical cancer detection rate.

\section{Background}

Cervical cancer is the third most common cancer among women worldwide and is the second most common to breast cancer in Asia ${ }^{[1]}$.Approximately $90 \%$ of cervical cancer deaths occur in developing countries, which is higher than that of developed countries ${ }^{[2]}$. Persistent high-risk human papillomavirus (hr-HPV) infection is main cause of cervical lesions ${ }^{[3,4]}$. At present, more than 200 genotypes of HPV have been isolated and the carcinogenicity of different HPV genotypes varies widely ${ }^{[5]}$. HPV 16 causes $60 \%$ of cancers and $50 \%$ of precancerous lesions, however, HPV56 rarely causes cancer ${ }^{[6-8]}$. It takes 20-30 years from precancerous lesions to cervical cancer, and such long period provides doctors possibilities for intervention ${ }^{[9,10]}$. Cervical cytology test and HPV test are the most common methods for cervical cancer screening.

Thinprep cytologic test (TCT) is a commonly used method for cytology test. According to the Bethesda system 2011 ${ }^{[11]}$, atypical squamous cells of undetermined significance (ASC-US) is an equivocal category, and the diagnosis is subjectively affected and poorly repeatable ${ }^{[12]}$. ASC-US account for approximately $3-5 \%$ of cytology results in cervical screening of asymptomatic women ${ }^{[13,14]}$, and comprise a wide variety of cervical cells, almost covering all pathological results. ASC-US may be related to benign reactions, such as microbial infections and inflammation, and it may also be related to cervical lesions. There are a variety of histologic results among women with ASC-US ${ }^{[15]}$. However, only $7 \%-10 \%$ of ASC-US women was diagnosed as cervical intraepithelial neoplasia $(\mathrm{CIN}) 3$, and there was very low probability of invasive carcinoma for ASC-US women ${ }^{[16]}$.

There are three common HPV tests in China. The Cobas 4800 HPV test performs DNA extraction, Polymerase Chain Reaction amplification, and real time detection by an automated sample preparation, to detect $14 \mathrm{hr}-\mathrm{HPV}$ genotypes. The HPV GenoArray test simultaneous identification of 21 individual HPV genotypes and efficient detection of single or multiple HPV infection, which preforms DNA amplification and HybriBio's proprietary flow through hybridization technique. Hybrid Capture 2 test $(\mathrm{HC} 2)$ is one of the most frequently applied test to detect the presence of any $13 \mathrm{hr}-\mathrm{HPV}$ types, which can provide a quantitation of the viral DNA load. 
This study aimed to investigate the distribution of hr-HPV genotypes, the association between the severity of the cervical lesions and hr-HPV viral loads, and age-stratified prevalence of high-grade squamous intraepithelial lesion (HSIL) and cervical cancer, by analyzing of clinical information of HPV-positive/ASC-US women in China, which would help us find an appropriate triage of women with ASC-US cytology in the future.

\section{Methods}

\section{Study population}

After approval of the Qilu Hospital of Shandong University, this retrospective study included women who accepted TCT test, HPV test and colposcopy at Qilu Hospital of Shandong University from January 2012 to January 2019.

Inclusion criteria: (1) women with ASC-US cytology and hr-HPV infections; (2) women accepted colposcopic examination and underwent cervical biopsy under colposcopic guidance; (3) women with complete cervical cervix.

Exclusion criteria: (1) women with history of treatments to cervical lesions, such as cervical surgery, laser, freezing and medication, et al.; (2) women with malignant tumors such as ovarian cancer and endometrial cancer; (3) women with autoimmune diseases or receiving immunotherapy; (4) women with pregnancy; (5) women who had received the HPV vaccine; (7) women with smears of insufficient quality (with an absence of endocervical cells).

\section{Cytology test and HPV Test}

No vaginal washing, intravaginal medication or physical therapy for 3 days before the samples were collected. No sexual behavior within 24 hours and women were in non-menstrual period when the samples were collected. Specimen collection, specimen preparations, and results of test are performed, according to the instructions of the manufacturers, respectively.

ThinPrep (Hologic Inc, Marlborough, Massachusetts, USA囚preparations was used and cytological diagnosis was performed by experienced cytopathologists, according to the Bethesda system $2011^{[11]}$.

Women could choose one of three HPV tests:

(1) HC2 (Qiagen, Gaithersburg, MD, USA): semi-quantitative detection of 13 hr-HPV types: 16, 18, 31, 33, 35, 39, 45, 51, 52, $56,58,59$ and 68. Positive by the ratio relative-light-unit/cut-off (RLU/CO) was $>1.0$ (equivalent to $1.0 \mathrm{pg} \mathrm{HPV} \mathrm{DNA/mL} \mathrm{or}$ 100000 HPV copies/mL).

(2) the Cobas 4800 System (Roche Diagnostics Corporation, Indianapolis, Indiana): Qualitative detection of HPV 16, HPV 18 and 12 hr-HPV (a pool of 12 other hr-HPV types: including HPV 31,33,35,39,45,51,52,56,58,59,66 and 68).

(3) the HPV GenoArray test (Hybribio Biotechnology Ltd Corp, Chaozhou, Guangdong Province, China): qualitative detection of 15 kinds of hr-HPV types: 16, 18, 31, 33, 35, 39, 45, 51, 52, 53, 56, 58, 59, 66 and 68; 6 kinds of low risk HPV (Ir- HPV) types: 6, 11, 42, 43, 44 and 81(CP8304).

\section{Colposcopy and Guided Cervical Biopsies}

All women, enrolled in this study, underwent visual inspection with acetic acid and insulin inspection with Lugo's iodine (VILI) and colposcopy. If any of suspicious lesion were found among the above mentioned examinations, multi-point biopsy was taken directed by colposcopy at the suspected lesion; otherwise, a random four-quadrant cervical biopsy was obtained at the squamocolumnar junction. Women who were not satisfied with colposcopy should underwent Endocervical Curettage, if necessary. 
The histologic diagnosis was based on the consensus diagnosis of two experienced gynecologic pathologists. All gynecologic pathologists were blinded to results of TCT, HPV test and colposcopic examination. The results of biopsy reports include: Normal; low-grade squamous intraepithelial lesion (LSIL) (including CIN 1), high-grade squamous intraepithelial lesion (HSIL) (including CIN 2-3), and cervical cancer. HSIL or worse (HSIL+) includes HSIL and cervical cancer. The gold standard for this study was the histologic diagnosis.

\section{Statistical Analysis}

Statistical analyses were performed using the SAS 9.4 (SAS Institute, Cary, NC) for windows. The categorical variables were expressed by a percentage (\%). Cochran-Armitage Trend test was used to analyze the the detection rate of HSIL+ in different viral load groups. The binary Logistic regression model was used to analyze the detection rate of HSIL+ and cervical cancer in different age groups. Results were considered statistically significant at p-values $<0.05$.

\section{Results}

\section{Characteristics of the study population}

Clinical information of 250,000 women who underwent HPV and TCT test at Qilu Hospital of Shandong University from January 2012 to January 2019 was retrospectively collected and analyzed. Among them, 7001(2.80\%) women were diagnosed as ASC-US by TCT test, including 3459 (49.41\%) women with hr-HPV infection and 3542 (50.59\%) women without hr-HPV infection. Finally, a total of 3,459 ASC-US women with hr-HPV infection were enrolled in this study finally.

A total of 1973 women chose the HPV typing test, identified the specific types of hr-HPV they were infected with, among whom 376 were 12 hr-HPV positive (from Cobas 4800 HPV test), 1,043 women had single HPV infection, and 554 women had multiple HPV infections. 1245 women were diagnosed with hr-HPV positive by HC2 test. The remaining hr-HPV positive 241 women who lost their results of HPV test, were classified as ANY group in this study. (We did not know the specific genotypes, nor did we know whether it is a single infection or multiple infections. We only knew that they were hr-HPV positive by their medical records during this study period.)

The average age of women was 39.86 \pm 9.94 years (range: $15-80$ years). The median number of pregnancies was 3 (range: $0-14$ ) and the median number of births was 1 (range: $0-8)$. This study divided the age into 5 age groups $\leq 30$ ( $n=654$, $18.91 \%), 31-40(n=1258,36.37 \%), 41-50(n=1011,29.23 \%), 51-60(n=448,12.95 \%)$, and $\varangle 60(n=88,2,54 \%)$.

\section{Distribution of histological results among different genotypes in ASC- US women}

The common hr-HPV genotype were shown in Table 1 and Figure 1, in women with ASC-US. In addition, the 31-40-year-old group $(n=1243,35.94 \%)$ and $40-49$-year-old group $(n=1087,31.43 \%)$ account for $65.6 \%$ of the total group.

This study calculated the rate of HSIL+ detection in women with HPV16, 18, 33, 39, 51, 52, 53, 56, 58, and 66 single infection (Table 2). And, other hr-HPV genotypes (except HPV 16, 18, 33, 39, 51, 52, 53, 56, 58, and 66) were grouped into the group of "others" due to the small number of people included in women with the single hr-HPV infection. The HSIL+ detection rates were different in different hr-HPV genotypes, and the rates of HSIL+ ranged from $8.51 \%$ to $63.09 \%$ in ASC-US women with single hr-HPV infection. The HSIL+ detection rate of women with single HPV16 infection (63.09\%) was the highest, followed by HPV33(57.50\%), HPV51(36.11\%), HPV58(36.11\%), HPV52(28.28\%), HPV18(26.37\%), HPV66(19.35\%), HPV39 (18.92\%), HPV53(15.00\%), and HPV56(8.51\%). The HSIL+ detection rate in the "others" group was 30.09\% (Table 2). Overall, a total of 1403 women had single HPV infection, of which 414(29.51\%) were diagnosed as HSIL and 22(1.57\%) 
women were diagnosed as cervical cancer by histological test, and the rate of HSIL+ in women with single HPV infection was $31.08 \%$.

Among women with the multiple infections, the HSIL+ detection rates in women with double hr-HPV infections, triple hr-HPV infections, and quadruple or more hr-HPV infections were $39.77 \%, 41.49 \%$, and $45.00 \%$, respectively (Table 2).

For those the specific hr-HPV types could not be identified, the detection rate of HSIL+ in ASC-US women with $12 \mathrm{hr}$-HPV test positive result (detected by Cobas $4800 \mathrm{HPV}$ ) was $32.45 \%$, and the detection rate of HSIL+ in ASC-US women with hrHPV positive results was $30.35 \%$ (Table 2 ).

A total of $45(1.30 \%)$ of the women in this study were diagnosed with cervical cancer, including 24 women with HPV16 infections (including 19 women with HPV16 single infection and 5 women with HPV16 multiple infections), 3 women with HPV18 single infection, 1 woman with HPV33 single infection, 1 woman with 12 hr- HPV infection (from Cobas 4800 HPV test), and 16 women with unidentified hr-HPV genotypes infections ( 11 women were HC2 positive, and 5 women belonged to ANY group).

\section{Distribution of histological results among viral load groups in ASC-US women}

1245 women tested positive for hr-HPV by HC2 test. $42.93 \%(n=398)$ of women with ASC-US had normal histological results, $22.76 \%(n=211)$ had LSIL, 33.23\% $(n=308)$ had HSIL, and 1.08\% $(n=308)$ had cancer. $34.30 \%(n=318)$ of women were diagnosed with HSIL+. According to the viral load of hr-HPV, women were divided into 3 groups: low viral load group ( $1 \leq R L U / C O<10, n=189)$, medium viral load group $(10 \leq R L U / C O<100, n=212)$, and high viral load group (RLU/CO $\geq 100, n=526)$.

The pathological distribution of these three viral-load groups was shown in Table 3. The percentage of women with normal pathology in the low-viral-load group (65.61\%) was highest, followed by intermediate-viral-load group (41.98\%) and highviral-load group (35.17\%). However, detection rate of HSIL+ in low-viral-load group was lowest, followed by intermediateviral-load group (34.91\%) and high-viral-load group (40.68\%), and there was is statistically significant between detection rate of HSIL+ and hr-HPV viral load $\left(\chi^{2}=35.03, P \otimes 0.0001\right)$.

\section{Distribution of histological results among age groups in ASC-US women}

Overall, 43.51\% ( $n=1505)$ of women with ASC-US had normal histological results, $20.84 \%(n=721)$ had LSIL, $34.35 \%(n=$ 1188) had HSIL, and $1.30 \%(n=45)$ had cervical cancer. The rate of HSIL+ of hr-HPV-positive/ASC-US women was $35.65 \%$ (Table3). Age-stratified prevalence of HSIL+ and cervical cancer in hr-HPV-positive/ASC-US women is shown in Figure 2.

The HSIL+ detection rate of $\leq 30$-year-old age group (40.52\%) was the highest; however, the detection rate of HSIL+ (21.65\%) was the lowest in the 51-60-year-old group. The HSIL+ detection rates of other age groups were $39.67 \%$ for group of women aged $31-40$ years, $34.22 \%$ for group of women aged $41-50$ years, and $29.55 \%$ for group of women aged $>60$ years. The women in $\leq 30(\mathrm{OR}=2.465 ; 95 \% \mathrm{Cl}$ : 1.875-3.241), 31-40 (OR=2.379; 95\% Cl: 1.850-3.060), 41-50 (OR = 1.883; $95 \% \mathrm{Cl}: 1.452-2.441$ ) year-old groups had significantly higher risk of HSIL+ compared with those in 51-60-year-old

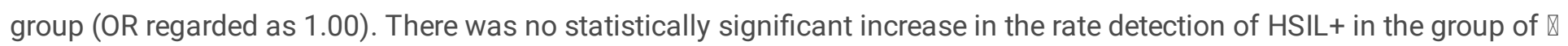
60 years by $36.49 \%$ (29.55\% vs $21.65 \%$; OR $=1.517 ; 95 \% \mathrm{Cl}$ : $0.911-2.527)$, compared with the $51-60$-year-old group (Table 4).

The cervical cancer detection rates were $0.61 \%, 0.95 \%, 1.38 \%, 2.68 \%$ and $3.41 \%$, respectively, in group of women aged $\leq 30$ years, 31-40 years, $41-50$ years, $51-60$ years and $>60$ years. When regarding the rate of cervical cancer of women in $\leq 30-$ year-old group who had the lowest rate of cervical cancer as the compared baseline (OR as 1.00), the women in $\leq 51-60$

Page 5/15 
$(\mathrm{OR}=4.472 ; 95 \% \mathrm{Cl}: 1.433-13.957)$ and $>60(\mathrm{OR}=5.735 ; 95 \% \mathrm{Cl}: 1.262-26.064)$ year-old groups were at increased risk for cervical cancer. There was no statistically significant increase in the rates detection of cervical cancer in the group of $31-$ 40 years by $55.74 \%(0.95 \%$ vs $0.61 \%$; OR $=1.565 ; 95 \% \mathrm{Cl}$ : $0.503-4.872)$ and the group of $41-50$ years by $126.23 \%(1.38 \%$ vs $0.61 \%$; OR $=2.282 ; 95 \% \mathrm{Cl}$ : $0.748-6.963)$, compared with the $\leq 30$-year-old group (Table 4 ).

\section{Discussion}

Currently, the HPV vaccine is gradually being used in the developing countries, but it has not fully covered all the regions. The effect of the HPV vaccination will reveal after several decades. In addition, the cervical cancer screening is still an essential method to prevent and treat cervical cancer for older women. Therefore, it's significant to adopt the cervical cancer screening method to diagnose and prevent the cervical cancer.

ASC-US is an ambiguous term and an exclusionary diagnosis that suggests a risk of disease rather than a definitive diagnosis of abnormal lesions. For women with ASC-US, if the diagnosis is not clear or not timely, the optimal treatment will be delayed; on the other hand, the excessive diagnosis and treatment will not only bring physical and psychological burden to the women, but also can cause adverse pregnancy outcomes ${ }^{[17,18]}$. In addition, it will increase the family and society economic burden and will not allocate medical resources reasonably.

This retrospective cross-sectional single-center study in China, lasting for 7 years, collected clinical data of 250,000 women who underwent HPV and TCT test at Qilu Hospital of Shandong University. The frequency of ASC-US was $2.80 \%(n=7001)$ in cervical screenings of this center, which was suggested the center strictly controlled the frequency of occurrence of ASCUS and reduced the phenomenon of ascus as the "Garbage Dump", according previous research [11]. Some studies have reported that the prevalence of CIN2+ among women with ASC-US is between $5 \%-39 \%[13,19,20]$. The detection rate of HSIL+ in hr-HPV-positive/ASC-US women in this study was $35.65 \%$, which was higher than some studies. The possible reasons are as follows: (1) The target population of this study was hr-HPV-positive/ASC-US women, while the target population of other studies were ASC-US women (including hr-HPV positive and negative women). The reported prevalence of hr-HPV among women with ASC-US in most studies was $23 \%$ to $74 \%{ }^{[21]}$. (2) This study was a retrospective study with the possibility of incomplete data collection.

In this cross-sectional study, the rates of HSIL+ ranged from $8.51 \%$ of HPV 56 to $63.09 \%$ of HPV16 in ASC-US women with single hr-HPV infection. The HSIL+ detection rates in women with HPV multiple infections was more than $39.77 \%$. Previous research reported HPV-positive/ ASC-US women would have similar the 2-year cumulative risk of HSIL+ and be clinically equivalent with women with LSIL Pap results ${ }^{[14]}$. We look forward to relevant longitudinal researches on relationship between HPV genotypes and risk of cervical lesions.

Systematic review ${ }^{[22-24]}$ and a large randomized trial ${ }^{[13]}$ consistently showed that, compared with repeat cytology, the accuracy of HC2 to detect underlying HSIL+ was higher in triage of women with ASC-US cytology. Previous studies suggested that the test may optimized by using a cutoff higher than this $1.0 \mathrm{pg} / \mathrm{mL}{ }^{[25-27]}$. The rates of HSIL+ in higher viral road of women with ASC-US cytology were higher (Cochran-Armitage Trend test $\chi^{2}=35.03, P \otimes 0.0001 \rrbracket$ in this study.

The highest detection rate of $\mathrm{HSIL}+(40.52 \%)$ was in the age group of women aged $\leq 30$ years, and the lowest detection rate of HSIL+ (21.65\%) was in the 51-60-year-old group in this study. A large longitudinal cohort study lasting 7 years within the Guanacaste population showed older women had a similar or slightly decreased risk of HSIL+ and especially CIN 3 compared with younger women ${ }^{[28]}$. In addition, a systematic review included in 103 studies (including more than

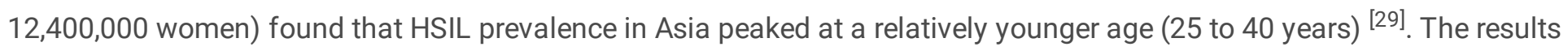
in these studies are in coincidence with our findings. When calculating the cervical cancer detection rate, this study showed that the value of the cervical cancer detection rate increased with age, and older women had higher cervical cancer detection rates than younger women. The reasons are as follows: 
(1) Due to hormone levels, the use of intrauterine device, more sexual behavior and inflammation in younger women, the cells of those with benign morphological changes mixed with cells of true precancerous lesions, and it may interfere doctors' diagnosis.

(2) HPV prevalence rate among younger women are higher, and the rate of HPV prevalence is highly age-related and decreases with age ${ }^{[30]}$. According to previous study, the HPV prevalence rate could be as high as $70 \%$ for women of aged $<25$ years $^{[31]}$.

(3) The previous researches found that CIN1 and a subset CIN2 lesions are clinical manifestations of the result of productive hr-HPV infection ${ }^{[32]}$, which can regularly regress within 1-2 years spontaneously and have a low risk to progress to invasive carcinoma ${ }^{[33]}$.

(4) Older women could clear newly HPV infections (including HPV16 infections) as quickly as younger women ${ }^{[28]}$. So, the lowest detection rate of $\mathrm{HSIL}+$ was in the group aged 51-60 years old.

(5) Lower estrogen levels, thinning of the epithelium, decreasing lactobacilli and some genital tract infections combined diabetes etc., create favorable conditions for HPV infection in older women, especially for $\$ 60$ years old women.

(6) Another subset of CIN2 lesions and CIN3 lesions are clinical manifestations of the result of transforming hr-HPV

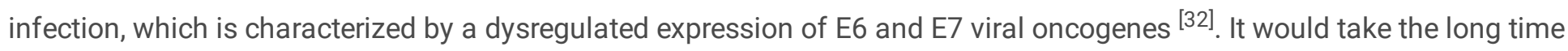
of 20-30 years for the progression to invasive carcinoma from precancerous lesion in most patients ${ }^{[10]}$.

(7) What's more, persistent hr-HPV infection is a risk factor for cervical cancer and the risk of persistence increases with the increasing age of the woman. Therefore, older ASC-US women have a higher cervical cancer detection rate.

However, this study showed there was no statistically significant increase in the rate detection of HSIL in the group of $₫ 60$ years by $36.49 \%$ (29.55\% vs $21.65 \%)$, compared with the $51-60$ years group, which may be related to the relatively limited sample we studied.

A 2013 Cochrane Meta-analysis studied triage ASC-US by repeat cytology versus HPV testing and found that similar pooled CIN2+ and CIN3+ specificities that likely will translate to similar overtreatment rates ${ }^{[16]}$. So, one way to solve the problem of overtreatment is to consider alternatives new method to triage women with ASC-US. Molecular markers may help identify cells with abnormal cell morphology. P16 or dual p16 and Ki-67 immunostaining on cytological preparations provides a promising method to triage HPV-positive women ${ }^{[34,35]}$. Biomarkers based on DNA methylation includes methylation markers representing various combinations of genes, such as SRY-box 1 (SOX1), PAX1, NK6 homeobox 1 (NKX6-1) [36], junctional adhesion molecule 3 (JAM3), EPB41L3, TERT, C130RF18 [37], and CADM1/MAL [38].

Combined with knowledge about the biology of CIN and results of this study, here are a few suggestions to triage women with ASC-US cytology. Firstly, we should ensure the quality of TCT test and strictly control the occurrence frequency of ASCUS. It has been suggested that good management requires that the frequency of ASC be maintained at $<5 \%$ of all cervical screenings ${ }^{[11]}$, and ASC:SIL ratio be maintained at $<1.5$. Secondly, ASC-US women with obvious inflammation should accept anti-inflammatory treatment, and repeat cytology test 4-6 months after inflammation disappearing, especially young women. If the cytological results of women $\geq A S C-U S$, then they are recommended to refer to colposcopy. In addition, we should strictly follow up on young women who have been diagnosed as HSIL by histological examination, rather than implementing excessive treatment. Finally, it is more and more important for us to find a new triage test.

\section{Conclusion}


ASC-US women with HPV 16/18/33/51/52/58 single infection and multiple infections, as well as high HPV viral loads, have high risk of $\mathrm{HSIL}+$. In addition, the rate of HSIL+ was higher and the rate of cervical cancer was lower in younger hrHPV-positive/ ASC-US women, in contrast with older women.

\section{Limitations}

This study was a retrospective single-center study with the possibility of incomplete data collection and limited data sources. What's more, women enrolled in this study were tested by there HPV tests and different HPV tests varied in sensitivity and specificity. Relatively large prospective studies were expected to confirm the findings of this study in the future.

\section{Abbreviations}

hr-HPV = high-risk human papillomavirus

TCT = Thinprep cytologic test

ASC-US = atypical squamous cells of undetermined significance

$\mathrm{CIN}=$ cervical intraepithelial neoplasia

HC2 $=$ Hybrid Capture 2 test

$\mathrm{RLU} / \mathrm{CO}=$ the ratio relative-light-units /cut-off

LSIL = low-grade squamous intraepithelial lesion

HSIL = high-grade squamous intraepithelial lesion

$\mathrm{HSIL}+=$ high-grade squamous intraepithelial lesion and cervical cancer

\section{Declarations}

\section{Ethics approval and consent to participate}

All procedures in studies involving human participants were performed in accordance with ethical standards and approved by the Ethics Committee of Shandong University [2018 (054)].

\section{Consent for publication}

The manuscript was approved for publication by all authors.

\section{Availability of data and material}

We declared that materials described in the manuscript, including all relevant raw data, will be freely available to any scientist wishing to use them for non-commercial purposes, without breaching participant confidentiality.

\section{Competing interests}

The authors declare that they have no competing interests. 


\section{Funding}

This work was supported by the foundation of National Natural Science Foundation of China and Science, Grant/Award Number: 81874105; National Clinical Research Center for Gynecological Oncology, Grant/Award Number:2015BAl13B05; National Natural Science Foundation of China and Science, Grant/Award Number: 81372809; and National Key Research \& Development Program of China, Grant/Award Number: 2016YFC1302903. The funding sources acknowledged the design of the study but did not influence the process of our study, including data collection, analysis, and interpretation and the reporting of results.

\section{Authors' contributions}

ZW designed the study and completed the initial draft of paper. YG, HW and JC conducted the data collection. YZ and LJ carried out the analysis of the data. $\mathrm{XY}$ and $\mathrm{BC}$ founded for the study. All authors read and approved the final manuscript.

\section{Acknowledgements}

The authors acknowledge all participants for their helpful cooperation. We thank Miss Mingming Wang from Shandong University for her intellectual support in data analysis.

\section{Authors' information}

Affiliations

Department of Obstetrics and Gynecology, Qilu Hospital, Shandong University, Jinan, China

Zhiling Wang, Ying Gu, Hui Wang, Junyu Chen, Yawen Zheng, Lin Jia, Baoxia Cui, \& Xingsheng Yang

\section{References}

1. Lertkhachonsuk AA, Yip CH, Khuhaprema T, Chen DS, Plummer M, Jee SH, et al. Cancer prevention in Asia: resourcestratified guidelines from the Asian Oncology Summit 2013. Lancet Oncology, 2013. 14(12): p. E497-E507.

2. .Torre LA, Bray F, Siegel RL, Ferlay J, Lortet-Tieulent J, Jemal A. Global Cancer Statistics, 2012. CA Cancer J Clin. 2015 Mar; 65(2):87-108.

3. .Nobbenhuis MA, Walboomers JM, Helmerhorst TJ, Rozendaal L, Remmink AJ, Risse EK,et al. Relation of human papillomavirus status to cervical lesions and consequences for cervical-cancer screening: a prospective study. Lancet. 1999 Jul 3;354(9172):20-5.

4. Group, I. M. W. IARC Monographs on the Evaluation of Carcinogenic Risks to Humans, No. 90: Human Papillomaviruses. Lyon (FR): International Agency for Research on Cancer, 2007: p. 1-670.

5. Choi YJ, Park JS. Clinical significance of human papillomavirus genotyping. J Gynecol Oncol. 2016 Mar;27(2):e21.

6. de Sanjose S, Quint WG, Alemany L, Geraets DT, Klaustermeier JE, Lloveras B, et al. Human papillomavirus genotype attribution in invasive cervical cancer: a retrospective cross-sectional worldwide study.Lancet Oncol. 2010 Nov;11(11):1048-56.

7. Guan P, Howell-Jones R, Li N, Bruni L, de Sanjosé S, Franceschi S, et al. Human papillomavirus types in 115,789 HPVpositive women: a meta-analysis from cervical infection to cancer. Int J Cancer. 2012 Nov 15;131(10):2349-59.

8. Bosch FX, Broker TR, Forman D, Moscicki AB, Gillison ML, Doorbar J, et al. Comprehensive control of human papillomavirus infections and related diseases. Vaccine. 2013 Dec 31;31 Suppl 7:H1-31. 
9. McCredie MR, Sharples KJ, Paul C, Baranyai J, Medley G, Jones RW et al. Natural history of cervical neoplasia and risk of invasive cancer in women with cervical intraepithelial neoplasia 3: a retrospective cohort study. Lancet Oncol. 2008 May;9(5):425-34.

10. Vink MA, Bogaards JA, van Kemenade FJ, de Melker HE, Meijer CJ, Berkhof J. Clinical Progression of High-Grade Cervical Intraepithelial Neoplasia: Estimating the Time to Preclinical Cervical Cancer From Doubly Censored National Registry Data. Am J Epidemiol. 2013 Oct 1;178(7):1161-9.

11. Solomon D, Davey D, Kurman R, Moriarty A, O'Connor D, Prey M, et al., The 2001 Bethesda System: terminology for reporting results of cervical cytology. JAMA. 2002 Apr 24;287(16):2114-9.

12. Davey DD, Neal MH, Wilbur DC, Colgan TJ, Styer PE, Mody DR. Bethesda 2001 implementation and reporting rates: 2003 practices of participants in the College of American Pathologists Interlaboratory Comparison Program in Cervicovaginal Cytology. Arch Pathol Lab Med. 2004 Nov;128(11):1224-9.

13. ASCUS-LSIL Traige Study (ALTS) Group. Results of a randomized trial on the management of cytology interpretations of atypical squamous cells of undetermined significance. Am J Obstet Gynecol. 2003 Jun;188(6):1383-92.

14. Katki HA, Schiffman M, Castle PE, Fetterman B, Poitras NE, Lorey T,et al. Five-Year Risks of CIN 3+ and Cervical Cancer Among Women With HPV Testing of ASC-US Pap Results. J Low Genit Tract Dis. 2013 Apr;17(5 Suppl 1):S36-42.

15. Cox JT, Schiffman M, Solomon D; ASCUS-LSIL Triage Study (ALTS) Group. Prospective follow-up suggests similar risk of subsequent cervical intraepithelial neoplasia grade 2 or 3 among women with cervical intraepithelial neoplasia grade 1 or negative colposcopy and directed biopsy. Am J Obstet Gynecol. 2003 Jun;188(6):1406-12.

16. Arbyn M, Roelens J, Simoens C, Buntinx F, Paraskevaidis E, Martin-Hirsch PP, et al., Human papillomavirus testing versus repeat cytology for triage of minor cytological cervical lesions. Cochrane Database Syst Rev. 2013 Mar 28; (3):CD008054.

17. Kyrgiou M, Koliopoulos G, Martin-Hirsch P, Arbyn M, Prendiville W, Paraskevaidis E. Obstetric outcomes after conservative treatment for intraepithelial or early invasive cervical lesions: systematic review and meta-analysis. Lancet. 2006 Feb 11;367(9509):489-98.

18. Arbyn M, Kyrgiou M, Simoens C, Raifu AO, Koliopoulos G, Martin-Hirsch P, et al. Perinatal mortality and other severe adverse pregnancy outcomes associated with treatment of cervical intraepithelial neoplasia: meta-analysis. BMJ. 2008 Sep 18;337:a1284.

19. Persson M, Elfström KM, Brismar Wendel S, Weiderpass E, Andersson S. Triage of HR-HPV Positive Women with Minor Cytological Abnormalities: A Comparison of mRNA Testing, HPV DNA Testing, and Repeat Cytology Using a 4-Year Follow-Up of a Population-Based Study. PLoS One. 2014 Feb 26;9(2):e90023.

20. Tropé A1, Sjøborg KD, Nygård M, Røysland K, Campbell S, Alfsen GC, et al. Cytology and Human Papillomavirus Testing 6 to 12 Months after ASCUS or LSIL Cytology in Organized Screening To Predict High-Grade Cervical Neoplasia between Screening Rounds. J Clin Microbiol. 2012 Jun;50(6):1927-35.

21. Del Mistro A, Frayle-Salamanca H, Trevisan R, Matteucci M, Pinarello A, Zambenedetti P, et al. Triage of women with atypical squamous cells of undetermined significance (ASC-US): Results of an Italian multicentric study. Gynecol Oncol. 2010 Apr;117(1):77-81.

22. Arbyn M, Buntinx F, Van Ranst M, Paraskevaidis E, Martin-Hirsch P, Dillner J. Virologic versus cytologic triage of women with equivocal Pap smears: a meta-analysis of the accuracy to detect high-grade intraepithelial neoplasia. J Natl Cancer Inst. 2004 Feb 18;96(4):280-93.

23. Arbyn M, Sasieni P, Meijer CJ, Clavel C, Koliopoulos G, Dillner J. Chapter 9: Clinical applications of HPV testing: a summary of meta-analyses. Vaccine. 2006 Aug 31;24 Suppl 3:S3/78-89.

24. Cuzick J, Arbyn M, Sankaranarayanan R, Tsu V, Ronco G, Mayrand MH, et al. Overview of human papillomavirus-based and other novel options for cervical cancer screening in developed and developing countries. Vaccine. 2008 Aug 19;26 Suppl 10:K29-41. 
25. Howard M, Sellors J, Kaczorowski J. Optimizing the Hybrid Capture II human papillomavirus test to detect cervical intraepithelial neoplasia. Obstet Gynecol. 2002 Nov;100(5 Pt 1):972-80.

26. Kulmala SM, Syrjänen S, Shabalova I, Petrovichev N, Kozachenko V, Podistov J, et al. Human papillomavirus testing with the hybrid capture 2 assay and PCR as screening tools. J Clin Microbiol. 2004 Jun;42(6):2470-5.

27. Lin HP, Huang YY, Wu HY, Kao JT. Method for testing for human papillomavirus infection in patients with cervical intraepithelial disease. J Clin Microbiol. 2004 Jan;42(1):366-8.

28. Rodríguez AC, Schiffman M, Herrero R, Hildesheim A, Bratti C, Sherman ME, et al. Longitudinal Study of Human Papillomavirus Persistence and Cervical Intraepithelial Neoplasia Grade 2/3: Critical Role of Duration of Infection. J Natl Cancer Inst. 2010 Mar 3;102(5):315-24.

29. Ting J, Kruzikas DT, Smith JS. A global review of age-specific and overall prevalence of cervical lesions. Int J Gynecol Cancer. 2010 Oct;20(7):1244-9.

30. Stoler MH, Wright TC Jr, Sharma A, Apple R, Gutekunst K, Wright TL, et al., High-risk human papillomavirus testing in women with ASC-US cytology: results from the ATHENA HPV study. Am J Clin Pathol. 2011 Mar;135(3):468-75.

31. Winer RL, Hughes JP, Feng Q, Xi LF, Cherne S, O’Reilly S, et al., Early Natural History of Incident, Type-Specific Human Papillomavirus Infections in Newly Sexually Active Young Women. Cancer Epidemiol Biomarkers Prev. 2011 Apr;20(4):699-707.

32. Steenbergen RD, Snijders PJ, Heideman DA, Meijer CJ. Clinical implications of (epi)genetic changes in HPV-induced cervical precancerous lesions. Nat Rev Cancer. 2014 Jun;14(6):395-405.

33. Mills AM1, Paquette C, Castle PE, Stoler MH. Risk Stratification By p16 Immunostaining of CIN1 Biopsies A Retrospective Study of Patients From the Quadrivalent HPV Vaccine Trials. Am J Surg Pathol. 2015 May;39(5):611-7.

34. Carozzi F, Gillio-Tos A, Confortini M, Del Mistro A, Sani C, De Marco L, et al. Risk of high-grade cervical intraepithelial neoplasia during follow-up in HPV-positive women according to baseline p16-INK4A results: a prospective analysis of a nested substudy of the NTCC randomised controlled trial. Lancet Oncol. 2013 Feb;14(2):168-76.

35. Petry KU, Schmidt D, Scherbring S, Luyten A, Reinecke-Lüthge A, Bergeron C, et al., Triaging Pap cytology negative, HPV positive cervical cancer screening results with p16/Ki-67 Dual-stained cytology. Gynecol Oncol. 2011 Jun 1;121(3):505-9.

36. Lai HC, Lin YW, Huang RL, Chung MT, Wang HC, Liao YP, et al. Quantitative DNA Methylation Analysis Detects Cervical Intraepithelial Neoplasms Type 3 and Worse. Cancer. 2010 Sep 15;116(18):4266-74.

37. Eijsink JJ, Yang N, Lendvai A, Klip HG, Volders HH, Buikema HJ, et al. Detection of cervical neoplasia by DNA methylation analysis in cervico-vaginal lavages, a feasibility study. Gynecol Oncol. 2011 Feb; 120, 280-283.

38. Overmeer RM, Louwers JA, Meijer CJ, van Kemenade FJ, Hesselink AT, Daalmeijer NF, et al. Combined CADM1 and MAL promoter methylation analysis to detect (pre-)malignant cervical lesions in high-risk HPV-positive women. Int J Cancer. 2011 Nov 1;129(9):2218-25.

\section{Tables}

Table 1. Distribution of hr-HPV genotypes in hr-HPV-positive/ASC-US women. 


\begin{tabular}{|c|c|c|c|c|c|c|c|c|c|c|c|c|c|c|c|c|}
\hline \multirow[t]{2}{*}{ Age } & \multicolumn{15}{|c|}{ hr-HPV types } & \multirow[t]{2}{*}{$\mathrm{N}^{*}$} \\
\hline & 16 & 18 & 31 & 33 & 35 & 39 & 45 & 51 & 52 & 53 & 56 & 58 & 59 & 66 & $\begin{array}{l}68 \\
\end{array}$ & \\
\hline$\leq 30$ & 155 & 37 & 13 & 22 & 4 & 16 & 4 & 22 & 36 & 15 & 18 & 39 & 10 & 14 & 12 & 654 \\
\hline $31-40$ & 253 & 70 & 29 & 29 & 11 & 23 & 9 & 46 & 72 & 46 & 33 & 70 & 17 & 31 & 29 & 1258 \\
\hline $41-50$ & 167 & 56 & 26 & 29 & 13 & 32 & 7 & 22 & 66 & 36 & 34 & 55 & 16 & 22 & 23 & 1011 \\
\hline $51-60$ & 80 & 27 & 8 & 9 & 1 & 15 & 1 & 9 & 32 & 12 & 24 & 27 & 9 & 18 & 6 & 448 \\
\hline 060 & 28 & 4 & 1 & 2 & 1 & 8 & 1 & 2 & 2 & 5 & 5 & 7 & 0 & 2 & 1 & 88 \\
\hline $\mathbf{N}^{\dagger}$ & 683 & 194 & 77 & 91 & 30 & 86 & 22 & 101 & 209 & 114 & 114 & 198 & 52 & 87 & 71 & 3459 \\
\hline
\end{tabular}

ASC-US = atypical squamous cells of undetermined significance

hr-HPV =high-risk human papillomavirus

* The total number of people with hr-HPV positive in the corresponding age groups and this table did not list all HPV infection types.

† The sum of women with the type-specific hr-HPV infection(s) and it would be counted multiple times if the person has multiple hrHPV infections.

Table 2. Distribution of oncogenic hr-HPV types and histological results among hr-HPV-positive/ASC-US women.

\begin{tabular}{|c|c|c|c|c|c|c|}
\hline \multirow[t]{2}{*}{ HPV Types } & \multicolumn{6}{|c|}{ Histological Results } \\
\hline & TOTAL & NORMAL(n,\%) & LSIL(n,\%) & HSIL(n,\%) & $\mathrm{CA}(\mathrm{n}, \%)$ & HSIL+(n,\%) \\
\hline HPV16 & 401 & $97(24.19)$ & $51(12.72)$ & $234(58.35)$ & $19(4.74)$ & 253(63.09) \\
\hline HPV18 & 91 & $42(46.15)$ & $25(27.27)$ & $21(23.08)$ & $3(3.30)$ & $24(26.37)$ \\
\hline HPV33 & 40 & $11(27.50)$ & $6(15.00)$ & $22(55.00)$ & $1(2.50)$ & $23(57.50)$ \\
\hline HPV39 & 37 & $17(45.95)$ & $13(35.14)$ & $7(18.92)$ & $0(0.00)$ & $7(18.92)$ \\
\hline HPV51 & 36 & $16(44.44)$ & $7(19.44)$ & $13(36.11)$ & $0(0.00)$ & $13(36.11)$ \\
\hline HPV52 & 99 & $48(48.48)$ & $23(23.23)$ & $28(28.28)$ & $0(0.00)$ & $28(28.28)$ \\
\hline HPV53 & 40 & $29(72.50)$ & $5(12.50)$ & $6(15.00)$ & $0(0.00)$ & $6(15.00)$ \\
\hline HPV56 & 47 & $33(70.21)$ & $10(21.28)$ & $4(8.51)$ & $0(0.00)$ & $4(8.51)$ \\
\hline HPV58 & 108 & $38(35.19)$ & $31(28.70)$ & $39(36.11)$ & $0(0.00)$ & $39(36.11)$ \\
\hline HPV66 & 31 & $16(51.61)$ & $9(29.03)$ & $6(19.35)$ & $0(0.00)$ & $6(19.35)$ \\
\hline other* & 113 & $49(43.36)$ & $30(26.55)$ & $34(30.09)$ & $0(0.00)$ & $34(30.09)$ \\
\hline 2 types $^{\dagger}$ & 440 & 161(36.59) & $104(23.64)$ & $170(38.64)$ & $5(1.14)$ & 175(39.77) \\
\hline 3 types ${ }^{\ddagger}$ & 94 & $34(36.17)$ & $21(22.34)$ & $39(41.49)$ & $0(0.00)$ & $39(41.49)$ \\
\hline$\geq 4$ types $^{\S}$ & 20 & $6(30.00)$ & $5(25.00)$ & $9(45.00)$ & $0(0.00)$ & $9(45.00)$ \\
\hline $12 \mathrm{hr}-\mathrm{HPV}^{\|}$ & 376 & $179(47.61)$ & $75(19.95)$ & $121(32.18)$ & $1(0.27)$ & $122(32.45)$ \\
\hline $\mathrm{HC} 2$ & 1245 & $637(51.16)$ & $250(23.24)$ & $347(27.87)$ & $11(0.88)$ & $358(28.76)$ \\
\hline $\mathrm{ANY}^{\Uparrow}$ & 241 & $92(38.17)$ & $56(23.24)$ & $88(36.51)$ & $5(2.07)$ & $93(38.59)$ \\
\hline $\mathbf{N}$ & 3459 & $1505(43.51)$ & $721(20.84)$ & $1188(34.35)$ & $45(1.30)$ & $1233(35.65)$ \\
\hline
\end{tabular}

ASC-US $=$ atypical squamous cells of undetermined significance

$\mathrm{HPV}=$ human papillomavirus

hr-HPV = high-risk human papillomavirus

LSIL = low-grade squamous intraepithelial lesion

HSIL $=$ high-grade squamous intraepithelial lesion 
$\mathrm{CA}=$ cervical cancer

HSIL+= high-grade squamous intraepithelial lesion or worse

*= single hr-HPV infection except HPV16, 18, 33, 39, 51, 52, 53, 56, 58 and 66

$\dagger=$ double infections with hr-HPV

$\ddagger=$ triple infections

- =4 or more than kinds hr-HPV infections

$\|=$ infection(s) with a pool of 12 other hr-HPV types (including HPV 31,33,35,39,45,51,52,56,58,59,66 and 68) that did not know the specific type of HPV (from Cobas 4800 HPV test)

I $=$ hr-HPV infection that did not know the specific type of HPV $\square$ from Cervista HPV HR (Hologic) or Hybrid Capture 2 test!

Table 3. Distribution of histological results among viral load groups in ASC-US women

\begin{tabular}{ccccccc}
\hline Viral Load & \multicolumn{5}{c}{ Histological Results } \\
\cline { 2 - 6 } & TOTAL & NORMAL (n,\%) & LSIL (n,\%) & HSIL (n,\%) & CA (n,\%) & HSIL+ (n,\%) \\
\hline $1 \leq$ RLU/CO 10 & 189 & $124(65.61 \%)$ & $35(18.52 \%)$ & $28(14.81 \%)$ & $2(1.06 \%)$ & $30(15.87 \%)$ \\
\hline $10 \leq$ RLU/CO 100 & 212 & $89(41.98 \%)$ & $49(23.11 \%)$ & $72(33.96 \%)$ & $2(0.94 \%)$ & $74(34.91 \%)$ \\
\hline RLU/CO $\geq 100$ & 526 & $185(35.17 \%)$ & $127(24.14 \%)$ & $208(39.54 \%)$ & $6(1.14 \%)$ & $214(40.68 \%)$ \\
\hline N & 927 & $398(42.93 \%)$ & $211(22.76 \%)$ & $308(33.23 \%)$ & $10(1.08 \%)$ & $318(34.30 \%)$ \\
\hline
\end{tabular}

ASC-US $=$ atypical squamous cells of undetermined significance

$\mathrm{RLU} / \mathrm{CO}=$ the ratio relative-light-units /cut-off

LSIL= low-grade squamous intraepithelial lesion

HSIL= high-grade squamous intraepithelial lesion

$\mathrm{CA}=$ cervical cancer

HSIL+= high-grade squamous intraepithelial lesion and cervical cancer

Table 4. Distribution of histological results among viral load groups in ASC-US women. 


\begin{tabular}{|c|c|c|c|c|c|c|c|c|}
\hline \multirow[t]{2}{*}{ AGE } & \multicolumn{8}{|c|}{ Histological Results } \\
\hline & TOTAL & NORMAL(n,\%) & LSIL(n,\%) & HSIL(n,\%) & $\mathrm{CA}(\mathrm{n}, \%)$ & CA:OR(95\%CI) & HSIL+(n,\%) & HSIL+:OR(95\%CI) \\
\hline$\leq 30$ & 654 & $253(38.69 \%)$ & $136(20.80 \%)$ & 261(39.91\%) & $4(0.61 \%)$ & 1.00 & $265(40.52 \%)$ & $2.465(1.875-3.241)$ \\
\hline $\begin{array}{l}31- \\
40\end{array}$ & 1258 & $500(39.75 \%)$ & $259(20.59 \%)$ & 487(38.71\%) & $12(0.95 \%)$ & $\begin{array}{c}1.565(0.503- \\
4.872)\end{array}$ & 499(39.67\%) & $2.379(1.850-3.060)$ \\
\hline $\begin{array}{l}41- \\
50\end{array}$ & 1011 & $464(45.90 \%)$ & 201(19.77\%) & $332(32.84 \%)$ & $14(1.38 \%)$ & $\begin{array}{c}2.282(0.748- \\
6.936)\end{array}$ & $346(34.22 \%)$ & $1.883(1.452-2.441)$ \\
\hline $\begin{array}{l}51- \\
60\end{array}$ & 448 & $240(53.57 \%)$ & $110(24.55 \%)$ & $85(18.97 \%)$ & $12(2.68 \%)$ & $\begin{array}{c}\text { 4.472(1.433- } \\
13.957)\end{array}$ & $97(21.65 \%)$ & 1.00 \\
\hline 060 & 88 & 48(54.55\%) & $15(17.05 \%)$ & $23(26.14 \%)$ & $3(3.41 \%)$ & $\begin{array}{c}5.735(1.262- \\
26.064)\end{array}$ & 26(29.55\%) & $1.517(0.911-2.527)$ \\
\hline $\mathbf{N}$ & 3459 & $1505(43.51 \%)$ & $721(20.84 \%)$ & $1188(34.35 \%)$ & $45(1.30 \%)$ & - & $1233(35.65 \%)$ & - \\
\hline
\end{tabular}

ASC-US= atypical squamous cells of undetermined significance

HPV= human papillomavirus

hr-HPV =high-risk human papillomavirus

LSIL $=$ low-grade squamous intraepithelial lesion

HSIL $=$ high-grade squamous intraepithelial lesion

$\mathrm{CA}=$ cervical cancer

HSIL+= high-grade squamous intraepithelial lesion and cervical cancer

OR=odds ratio

$\mathrm{CI}=$ confidence interval

\section{Figures}

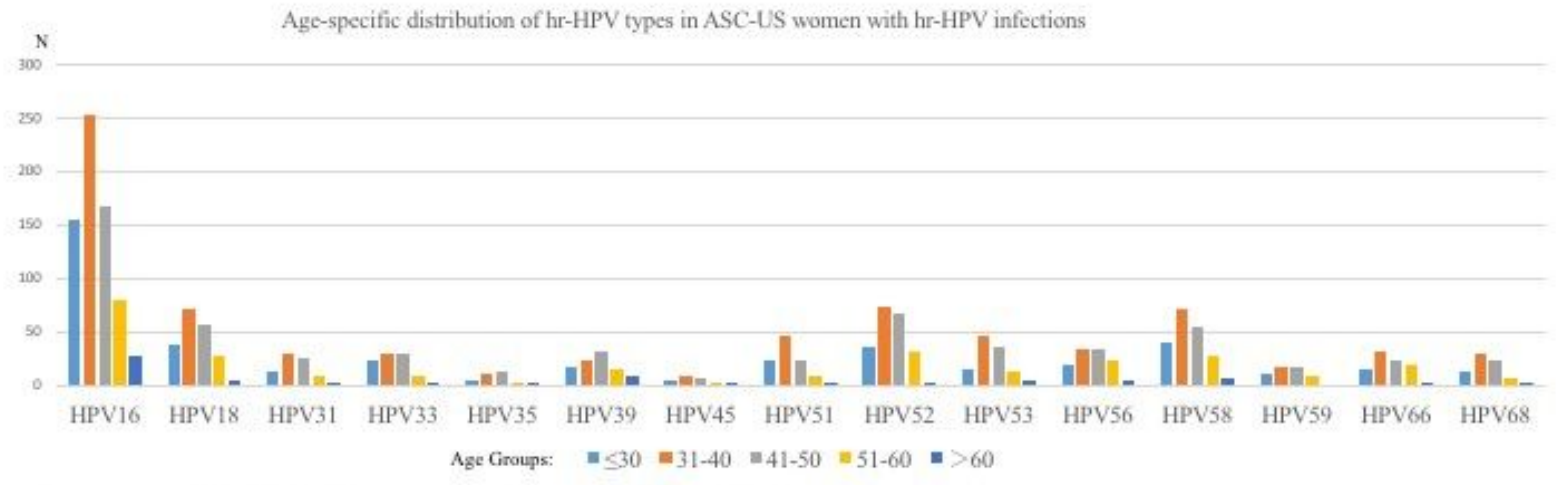

Figure 1. Age-specific distribution of hr-HPV types in ASC.US women with hr-HPV infections, hr-HPV: high-risk human papillomavirus

ASC.US: atypical squamous cells of undetermined significance

Figure 1 


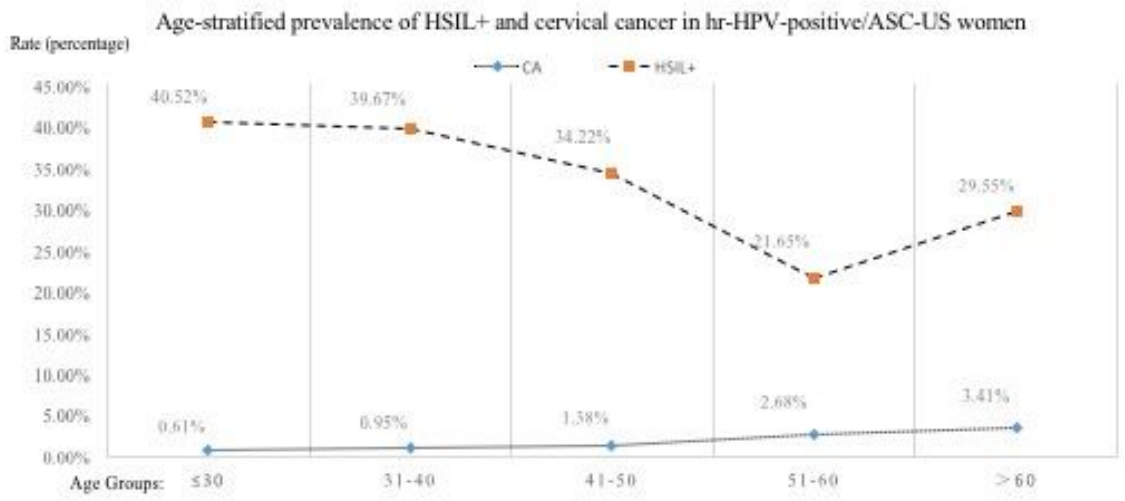

Figure 2. Age-stratified prevalence of HSIL+ and CA in hr-HPV-positive/ASC-US women.

HSIL. high-grade squamous intraepithelial lesion or worse

ASC.US: atypical squamous cells of undetermined significance

Figure 2

Page 15/15 